\title{
Twelve months follow-up after retrograde recanalization of superficial femoral artery chronic total occlusion
}

\author{
Joanna Wojtasik-Bakalarz, Salech Arif, Michał Chyrchel, Tomasz Rakowski, Krzysztof Bartuś, Dariusz Dudek, \\ Stanisław Bartuś
}

$2^{\text {nd }}$ Department of Cardiology and Cardiovascular Interventions, University Hospital, Krakow, Poland

Adv Interv Cardiol 2017; 13, 1 (47): 47-52

DOI: https://doi.org/10.5114/aic.2017.66186

\begin{abstract}
A bstract
Introduction: Fifty percent of cases of peripheral artery disease are caused by chronic total occlusion (CTO) of the superficial femoral artery (SFA). Ten-fifteen percent of percutaneous SFA recanalization procedures are unsuccessful. In those cases the retrograde technique can increase the success rate of the procedure, but the long-term follow-up of such procedures is still unknown.

Aim: To assess the efficacy and clinical outcomes during long-term follow-up after retrograde recanalization of the SFA.

Material and methods: We included patients after at least one unsuccessful percutaneous antegrade recanalization of the SFA. Patients were evaluated for the procedural and clinical follow-up of mean time 13.9 months.

Results: The study included 17 patients (7 females, 10 males) who underwent percutaneous retrograde recanalization of the SFA from June 2011 to June 2015. The mean age of patients was $63 \pm 7$ years. Retrograde puncture of the distal SFA was successful in all cases. A retrograde procedure was performed immediately after antegrade failure in 4 (23.5\%) patients and after a previously failed attempt in 13 (76.5\%) patients. The procedure was successful in 15 (88.2\%) patients, and unsuccessful in 2 (11.8\%) patients. Periprocedural complications included 1 peripheral distal embolization (successfully treated with aspiration thrombectomy), 1 bleeding event from the puncture site and 7 puncture site hematomas. During follow-up the all-cause mortality rate was $5.8 \%$ (1 patient, non-cardiac death). The primary patency rate at 12 months was $88.2 \%$ and secondary patency $100 \%$.

Conclusions: The retrograde SFA puncture seems to be a safe and successful technique for CTO recanalization and is associated with a low rate of perioperative and long-term follow-up complications.
\end{abstract}

Key words: peripheral artery disease, superficial femoral arteries, chronic total occlusion.

\section{Introduction}

Peripheral artery disease (PAD) affects more than $25 \%$ of the worldwide adult population over 65 years old, and the total number of patients with claudication increases with age [1]. More than $50 \%$ of cases of claudication are caused by chronic total occlusion (CTO) of the superficial femoral artery (SFA) [2]. Lesions causing total occlusions are usually longer and three times more common than lesions causing stenosis of the SFA [3]. The mean length of occlusions in the SFA is $20-40 \mathrm{~cm}$ [4]. From the pathophysiologic point of view, high risk plaques in the vessels of the lower limb are more fibrotic than plaques in coronary arteries and are composed of atheroma and organized thrombus [5].

Choosing the best treatment option for patients with PAD involves a decision between surgical bypass and an endovascular procedure. Based on the Trans-Atlantic
Inter-Society Consensus (TASC) II criteria, lesions categorized as class A-B are suitable for endovascular treatment, class $C$ lesions can be suitable for endovascular treatment, but surgery can be considered, and in class $\mathrm{D}$ lesions surgery should be taken into consideration [6]. There is a lack of studies showing direct comparison between these two methods with currently available stents, balloons and new operation techniques.

For the endovascular treatment, there are numerous new techniques to improve the effectiveness of the procedure. First of all, there are different approaches such as antegrade, transcollateral or retrograde. The retrograde access can be achieved via distal SFA, popliteal artery, tibial or transpedal arteries. To avoid bleeding complications, retrograde puncture is mostly performed under the control of ultrasound or fluoroscopy. In the group of patients with distal SFA/popliteal occlusion or with a nar-

\section{Corresponding author:}

Stanisław Bartuś MD, $2^{\text {nd }}$ Department of Cardiology and Cardiovascular Interventions, University Hospital, 36 Kopernika St, $31-501$ Krakow, Poland, phone: +48 1242471 74, e-mail: mbbartus@cyfronet.krakow.pl

Received: 3.03.2016, accepted: 28.08.2016. 
row lumen of tibial vessels the retrograde approach can be difficult. The most common complications are associated with bleeding events, perforations of the vessels or damage to the vascular-nervous bunch [7].

The access site is chosen based on type, location and morphology of the lesion, as well as the technique used for the previous attempts. In patients with unsuccessful antegrade recanalization the retrograde technique should be considered. In 10-15\% of unsuccessful SFA recanalization procedures the retrograde technique can increase the success rate of the procedure [8].

Secondly, the supreme challenge during the procedure is to reach the true lumen of the artery. Subintimal angioplasty uses hydrophilic wires to create the subintimal path and re-cross the distal lumen of the vessel [9].

In some centers re-entry devices such as Outback or Pioneer are available. The outcomes of crossing tools' usage are promising, but importantly they may increase the procedure cost. These tools are not widely available, require additional training and are not cost-effective [10-12].

Treatment of patients suffering from SFA CTO is still challenging, and there is a lack of current research showing long-term follow-up after retrograde recanalization of the SFA.

\section{Aim}

The aim of this retrospective study was to assess the efficacy and the clinical outcomes of retrograde recanalization of the SFA.

\section{Material and methods}

The data of all patients was collected in the $2^{\text {nd }}$ Cardiology Department, University Hospital in Krakow from June 2011 to June 2015. The study included 17 patients (7 females, 10 males) who underwent percutaneous retrograde recanalization of the SFA.

All patients selected for the retrograde procedures underwent at least one unsuccessful antegrade recanalization procedure of the target CTO lesion in the SFA. When the retrograde procedure was unsuccessful, rePTA (percutaneous transluminal angioplasty) with a retrograde approach or surgery was proposed to the patient.

Success of the procedure was defined as TIMI 3 and less than $30 \%$ of residual stenosis.

Patients were observed during hospitalization and 12 months after the procedure. Patients were also observed for perioperative complications (bleeding events) and damage to the nervous-vessel bundle and muscles around the distal puncture site.

Major adverse cardiac and cerebrovascular events (MACCE) during follow-up were defined as occurrence of all-cause death (cardiac and noncardiac), myocardial infarction, coronary revascularization, and stroke/temporary ischemic attacks (TIA).
Major adverse peripheral events (MAPE) during longterm follow-up were defined as occurrence of reintervention in the peripheral artery, or lower limb amputation.

\section{Periprocedural treatment}

Patients were treated by a combination of two antiplatelet drugs: aspirin $75 \mathrm{mg}$ and clopidogrel $75 \mathrm{mg}$ for 3 months after the procedure. After that time one of the drugs was discontinued. Additionally low-molecular-weight heparin was prescribed for 4 weeks. All patients received the maximum tolerated dose of statin. Other drug therapy was aimed at eliminating risk factors of atherosclerosis and treatment of comorbidities.

\section{Procedure}

On admission to our department all patients had coagulation parameters (APTT, PT) checked. Anticoagulation was adjusted to the weight of the patient. All patients signed written consent during admission to the hospital. The procedure required only local anesthesia.

All patients underwent angiography of the lower limb arteries during previous hospitalization or just before the retrograde procedure. Based on angiographic images the decision of retrograde access was made and the puncture was performed under the control of fluoroscopy. The patient was lying on the operating table in a standard position for angiography (on his back). The retrograde procedure required two approaches, proximal and distal, the patient remaining in a supine position during the whole procedure. The proximal approach is the puncture of the femoral artery in the groin area, usually contralateral, using $6 \mathrm{Fr}$ (mostly) vascular sheaths. The distal approach is the puncture of the SFA $5 \mathrm{~cm}$ above the knee joint, medially, with moderate flexion of the leg (needle: 12-15 cm, $21 \mathrm{G}$ ). In two cases 4 Fr vascular sheaths were needed to obtain proper support during the procedure. The retrograde puncture was obtained with fluoroscopic control and during contrast injections from the antegrade puncture. In a few cases with severe calcifications the puncture was facilitated by calcium. The puncture site was usually chosen in the reconnection area of the artery. Only in two cases was the retrograde puncture used for the distal part of the vessel. Through the antegrade approach a hydrophilic 0.035" guidewire (stiff, J-shaped; Terumo) was inserted. Occlusions were crossed from the retrograde approach with a non-hydrophilic 0.018" guidewire (soft V18 Boston Scientific). After predilatation using balloon catheters sized $2.5-4.0 \mathrm{~cm}$, in six cases stents were implanted from the antegrade approach (mean diameter: $6.5 \mathrm{~mm}$ and mean length: $144.5 \mathrm{~mm}$ ). Then postdilatation with balloon catheters (sized 5-6 cm, Biotronik) was performed. At the distal puncture site a cuff pressure manometer was used to maintain hemostasis. Antegrade vascular sheaths were left in the artery for $4 \mathrm{~h}$ after 
Table I. Demographic data and medical history of patients $(N=17)$

\begin{tabular}{|c|c|c|c|c|c|}
\hline Parameter & $N$ & Percent & Parameter & $N$ & Percent \\
\hline Gender (female/male) & $7 / 10$ & $41 / 59$ & Hyperlipidemia & 14 & 82.3 \\
\hline Age [years] & $63 \pm 7$ & - & Renal insufficiency & 2 & 11.7 \\
\hline Body mass index $\left[\mathrm{kg} / \mathrm{m}^{2}\right]$ & $28 \pm 5$ & - & Smoker & 14 & 82.3 \\
\hline Coronary artery disease & 14 & 82.3 & Previous coronary interventions & $8 \mathrm{PCl}, 2$ CABG & $47.3,11.7$ \\
\hline Hypertension & 15 & 88.2 & Previous stroke/TIA & 1 & 5.8 \\
\hline \multirow[t]{6}{*}{ Diabetes mellitus } & 10 & 58.8 & Previous unsuccessful PTA & 17 & 100 \\
\hline & & & Fontaine scale: & & \\
\hline & & & $2 a$ & 2 & 11.7 \\
\hline & & & $2 b$ & 14 & 82.3 \\
\hline & & & 3 & 1 & 5.8 \\
\hline & & & 4 & 0 & 0 \\
\hline
\end{tabular}

TIA - temporary ischemic attacks, PTA - percutaneous transluminal angioplasty.

the procedure and then removed. Then hemostasis was achieved by manual compression.

\section{Statistical analysis}

Results were presented as numbers (percentages) of patients or mean values \pm standard deviations where applicable. Additionally, survival free from cardiac and noncardiac death as well as free from MAPE was presented as Kaplan-Meier curves.

\section{Results}

\section{Patient population}

Cardiovascular disease was diagnosed in 14 (82.3\%) patients and 7 of them underwent acute coronary syndrome in the past (41.2\%). Percutaneous coronary interventions were performed in $8(47.0 \%)$ patients and coronary bypass grafting in $2(11.8 \%)$ cases.

According to data presented in Table I, all patients suffered from many comorbidities and can be considered as a group with high risk of cardiovascular complications.

\section{Angiographic data}

Chronic total occlusion was located in the right SFA in $47.0 \%$ and in the left SFA in $53.0 \%$.

In 15 cases standard antegrade (contralateral or ipsilateral) and retrograde (SFA) puncture were performed. In 2 other cases the puncture site was modified due to anatomical difficulties to brachial/popliteal artery puncture and femoral/ tibial anterior puncture. Unfractionated heparin was administered as an anticoagulant in 16 cases, while bivalirudin was used in 1 case.

Eight patients required one stent after recanalization, and 2 required two stents during one procedure. Average length of the stent was $144.5 \mathrm{~mm}$. Mostly drug-eluting balloons were used, with average length of the balloon measuring $135 \mathrm{~mm}$ (Table II).

\section{In-hospital observation}

Average time of hospitalization was 5.6 days, and average time from the procedure to discharge was 3.3 days. All patients admitted to our department presented symptomatic PAD of the lower limbs (mostly $2 b$ in Fontaine's scale).

Retrograde puncture was successful in all cases. Retrograde procedure was performed during the same index hospital stay, after antegrade failure in $4(23.5 \%)$ patients and after a previously failed attempt in 13 (76.5\%) patients.

The PTA procedure was successful in 15 (88.2\%) patients (TIMI 3 flow), and unsuccessful in 2 (11.8\%) patients during the first attempt. In those 2 patients the successful procedure was repeated after 4-6 weeks. Finally all 17 patients underwent full recanalization without the need for further surgical intervention (Table III).

Table II. Angiographic characteristics of treatment of CTO recanalization

\begin{tabular}{lcc} 
Parameter & $\mathbf{N}$ & Percent \\
\hline Artery: RSFA/LSFA & $8 / 9$ & $47 / 53$ \\
\hline $\begin{array}{l}\text { Stenting during PTA: } \\
\text { Diameter [mm] }\end{array}$ & 5,6 or 7 & \\
\hline Length [mm] & $144.5 \pm 44.6$ & 58.8 \\
\hline Number of stents & 10 & 88.2 \\
\hline Number of DEB & 15 & \\
\hline Average length of DEB [mm] & $135 \pm 21$ &
\end{tabular}

CTO - chronic total occlusion, DEB - drug-eluting balloon. 
Table III. In-hospital complications

\begin{tabular}{lcc}
\hline Parameter & N & Percent \\
\hline Death & 0 & - \\
\hline Artery perforation & 0 & - \\
\hline Distal embolization & 1 & 5.8 \\
\hline Puncture site bleeding & 1 & 5.8 \\
\hline Puncture site hematoma & 7 & 41 \\
\hline Proximal/distal & $4 / 3$ & $23.5 / 17.6$ \\
\hline Pseudoaneurysm & 0 & - \\
\hline $\begin{array}{l}\text { Damage to the nervous-vessel } \\
\text { bundle and muscles }\end{array}$ & 0 &
\end{tabular}

\section{Complications}

During hospitalization only a few complications occurred:

- 1 peripheral distal embolization, which was treated successfully with aspiration thrombectomy - the patient was discharged 3 days after procedure without the need of prolonged hospitalization after the adverse event;

- 1 case of bleeding from the proximal puncture site, which did not require surgical intervention or blood transfusion;

- 7 puncture site hematomas: 4 proximal and 3 distal. In all cases full muscle function and lack of neurological deficits were reported.

\section{Long-term follow-up}

During a mean follow-up of $13.9 \pm 4.0$ months the all-cause mortality rate was $5.8 \%$ (1 patient, non-cardiac death) (Figure $1 \mathrm{~A}$ ).

No myocardial infarction, urgent coronary revascularization or cerebrovascular events were observed.

A

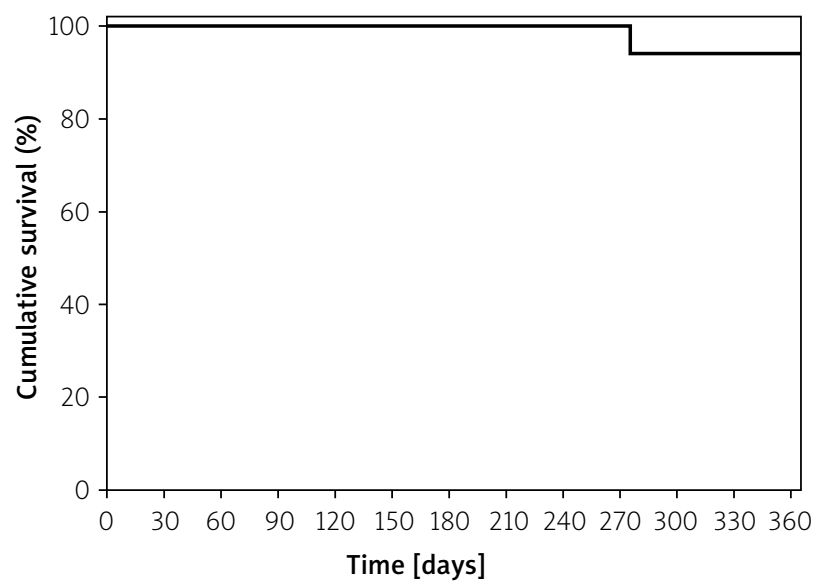

A PTA procedure of the same vessel was required in $2(11.7 \%)$ cases and a procedure of another vessel in $5(29.4 \%)$ cases, with average time to another procedure of 69.6 days. Indications for the procedures were established according to patients' symptoms and ultrasound.

The primary clinical patency rate of the recanalized artery at 12 months was $88.2 \%$. No late vascular complications were observed. No amputations were reported. The Kaplan-Meier curve for MAPE is shown in Figure $1 \mathrm{~B}$.

\section{Discussion}

We confirm that the retrograde approach for unsuccessful CTO recanalization in SFA can be safe and effective. Long-term follow-up of such patients shows a lack of serious cardiac and cerebrovascular events and efficacy of retrograde techniques with clinical patency at the rate of $88.2 \%$.

The PAD can be treated both surgically and endovascularly. Long lesions are challenging for the endovascular method, but the development of intraluminal techniques and devices changed the outlook on the treatment of peripheral arteriosclerosis [13]. The structure of the lesion can determine the success of recanalization. A harder, calcified part in the proximal section and softer in the distal section may be more successful in transluminal intervention of CTOs by the retrograde approach [14-16].

A disadvantage of the retrograde technique described in the literature was the need to change the position of the patient (supine-prone supine) during the procedure. This maneuver could result in dislocation of the femoral sheath, prolongation of the procedure or deterioration of respiratory function $[13,14,16]$. Modifications to the method allow one position of the patient to be maintained and complications associated with the procedure to be reduced [14, 17-19].

Now retrograde recanalization is associated with a low rate of serious complications between $2.5 \%$ and

B

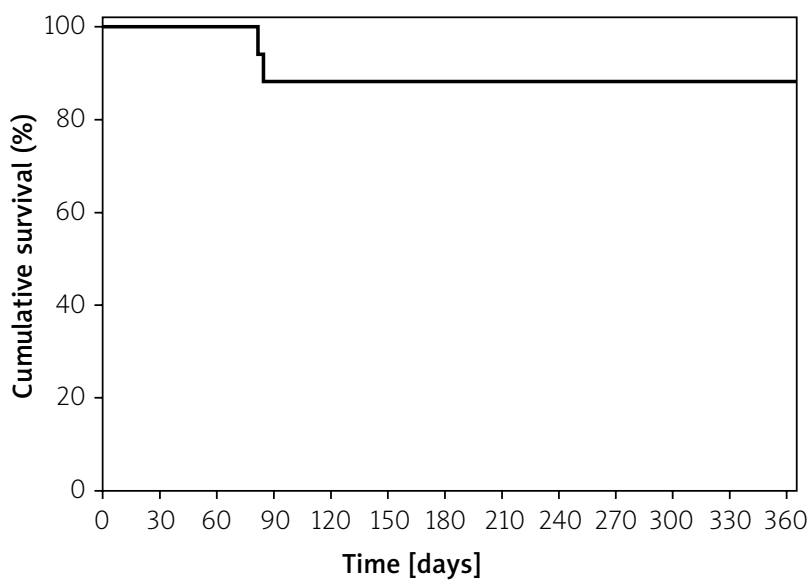

Figure 1. A - Kaplan-Meier curve for cardiac and non-cardiac death, B - Kaplan-Meier curve for major adverse peripheral events (MAPE) 
$5.2 \%[20,21]$. The most common are puncture site hematoma, arteriovenous fistula, pseudoaneurysm, and damage of the vascular-nervous bunch. In our study the rate of non-severe puncture site complications was $41 \%$ (7 puncture site hematomas, 4 proximal and 3 distal), and none of them caused prolonged hospitalization.

According to Sidhu et al. subintimal angioplasty in CTO SFA is associated with a $91 \%$ rate of technical success but only $73 \%$ of one-year primary patency [22]. Kim et al. give less optimistic data of primary patency at the rate of $52 \%$. There were no relevant complications described in that report [23].

The technical success rate of the outback re-entry system during PTA of SFA CTO ranges between $88 \%$ and $96 \%$. Occurrence of perioperative complications associated with access site was $4.8 \%$ for perforation of the target vessel and 5.0\% for hematoma and pseudoaneurysm. There was one reported case of a severe bleeding complication (fatal myocardial infarction associated with bleeding) $[10,11]$.

Mohamed El-Maadawy et al. reported technical success of the retrograde technique (prone or supine position) in 14 patients (82/4\%) with 2 dissections of the SFA, 1 popliteal artery aneurysm and 1 popliteal hematoma [24].

According to Noory et al., in retrograde transpopliteal access to CTO SFA, the primary success rate of the procedure was $98 \%$ with $10.7 \%$ of perioperative complications. The one-year restenosis rate was 55\% [21].

Schmidt et al. reported data on the retrograde approach in 50 patients. In their study the perioperative complication rate was $8 \%$, mostly associated with puncture site (4 pseudoaneurysms, 1 peripheral embolization and 1 arteriovenous fistula) [18]. However, up to now there is no other report showing the 12-month follow-up after such a procedure.

According to the American guidelines in PAD treatment the procedural success of femoral artery revascularization is assessed at $75-90 \%$ with a patency rate of $26-80 \%$ in 12 -month follow-up. In more than $50 \%$ of cases another procedure is required during 1-year follow-up $[25,26]$.

Some researchers showed the benefits of transpedal access after antegrade failure of CTO recanalization. Amoroso et al. performed a few recanalizations of SFA CTO via transpedal artery access with $100 \%$ success and without reported serious adverse events. In one-month follow-up all vessels were patent in ultrasound evaluation [27]. In the study by Ruzsa et al. 51 patients with critical limb ischemia due to occlusion in vessels below the knee were treated by retrograde transpedal recanalization. The success rate of revascularization was $78.4 \%$ with one major and three minor complications reported [28].

As compared to above-mentioned studies, especially the Schmidt et al. paper, in our study the rate of local, mild complications was higher, with a comparable rate of procedural complications. We reported all hematomas of the access site, including a subclinical one. The retrograde puncture was successful in all cases. In one case, due to severe calcifications, the distal access site was modified to tibial anterior artery puncture, with a good outcome and no severe complications. In our study the procedural success $(88.2 \%)$ and 1 -year patency rate $(88.2 \%)$ were higher than average data reported [16]. Patients in our group were burdened with many comorbidities, and all of them were considered for a retrograde approach after failed antegrade crossing. However, long-term follow-up did not reveal a significant rate of MACCE. The rate of major peripheral events was also satisfactory.

It is worth pointing out that in methods of recanalization of CTOs of the SFA there are wide differences in cost based on technology used during the procedure. Among the most expensive there are devices such as Outback LTD with costs of around $\$ 2,000$ or the even more expensive Pioneer catheter costing more than $\$ 3,000$ (requiring an IVUS machine) [12]. In the retrograde approach additional needles, wires and low profile balloons are unnecessary, which means total costs of no more than USD 100-200 [29].

\section{Limitations of the study}

It was a retrospective, single-center study. During the procedure activated clotting time was not checked. Clinical data based on medical history of hospitalization and data from long-term follow-up were collected in the outpatient clinical review. The studied group was small, and follow-up was limited to 12 months.

\section{Conclusions}

Retrograde SFA puncture is a safe and successful technique for CTO recanalization and is associated with a low rate of severe perioperative and long-term complications. The supine position of the patient during the procedure can decrease the rate of intraprocedural complications. This method should be considered for all patients after failure of antegrade crossing, but should be performed in experienced centers.

\section{Conflict of interest}

The authors declare no conflict of interest.

\section{References}

1. Norman PE, Eikelboom JW, Hankey GJ. Peripheral arterial disease: prognostic significance and prevention of atherothrombotic complications. Med J Aust 2004; 181: 150-4.

2. Nadal LL, Cynamon J, Lipsitz EC, et al. Subintimal angioplasty for chronic arterial occlusions. Tech Vasc Interv Radiol 2004; 7: 16-22.

3. Chopra P. Use of the VIABAHN ${ }^{\circledast}$ stent graft for PVD in the femoropopliteal arterial segment. One center's experience in 60 patients. Achieving success in the SFA. Supplement to Endovascular Today 2005; 4-7. 
4. Lammer J, Zeller T, Hausegger KA, et al. Heparin-bonded covered stents versus bare-metal stents for complex femoropopliteal artery lesions: the randomized VIASTAR trial (Viabahn endoprosthesis with PROPATEN bioactive surface [VIA] versus bare nitinol stent in the treatment of long lesions in superficial femoral artery occlusive disease). J Am Coll Cardiol 2013; 62: 1320-7.

5. Serrano Hernando FJ, Conejero AM. Peripheral artery disease: pathophysiology, diagnosis and treatment. Rev Esp Cardiol 2007; 60: 969-82.

6. Norgren L, Hiatt WR, Dormandy JA, et al. Inter-Society Consensus for the Management of Peripheral Arterial Disease (TASC II). J Vasc Surg 2007; 45 Suppl. S: S5-67.

7. Ueshima D, Ashikaga T, Shimura T, et al. Popliteal retrograde approach is effective and safe for superficial femoral artery chronic total occlusion. Ann Vasc Dis 2015; 8: 220-6.

8. Tai Z. Transcollateral approach for percutaneous revascularization of complex superficial femoral artery chronic total occlusion. J Invasive Cardiol 2013; 25: E96-100.

9. Geronemus AR, Peña CS. Endovascular treatment of femoralpopliteal disease. Semin Interv Radiol 2009; 26: 303-14.

10. Beschorner U, Sixt S, Schwarzwälder U, et al. Recanalization of chronic occlusions of the superficial femoral artery using the Outback re-entry catheter: a single center experience. Catheter Cardiovasc Interv 2009; 74: 934-8.

11. Aslam MS, Allaqaband S, Haddadian B, et al. Subintimal angioplasty with a true reentry device for treatment of chronic occlusion of the arteries of the lower extremity. Catheter Cardiovasc Interv 2013; 82: 701-6.

12. Smith M, Pappy R, Hennebry TA. Re-entry devices in the treatment of peripheral chronic occlusions. Tex Heart Inst J 2011; 38: 392-7.

13. George JC. Revascularization of superficial femoral artery chronic total occlusion. Vasc Dis Management 2012; 9: E48-50.

14. H'ng MWC, Punamiya S. An innovative modification of the retrograde approach to angioplasty and recanalization of the superficial femoral artery. Diagn Interv Radiol 2014; 20: 164-7.

15. Fanelli F, Lucatelli P, Allegritti M, et al. Retrograde popliteal access in the supine patient for recanalization of the superficial femoral artery: initial results. J Endovasc Ther 2011; 18: 503-9.

16. Lee HJ, Park SW, Chang IS, et al. Strategies for successful percutaneous revascularization of chronic total occlusion of the femoropopliteal arteries when the antegrade passage of a guide Wire Fails. Korean J Radiol 2012; 13: 467-75.

17. Kawarada O, Yokoi Y. Retrograde. 3-French popliteal approach in the supine position after failed antegrade angioplasty for chronic superficial femoral artery occlusion. J Endovasc Ther 2010; 17: 255-8.

18. Kawarada O. Miniaturized retrograde popliteal approach in a supine patient. J Endovasc Ther 2011; 18: 510-2.

19. Schmidt A, Bausback Y, Piorkowski M, et al. Retrograde recanalization technique for use after failed antegrade angioplasty in chronic femoral artery occlusions. J Endovasc Ther 2012; 19 23-9.

20. Yilmaz S, Sindel T, Ceken K, et al. Subintimal recanalization of long superficial femoral artery occlusions through the retrograde popliteal approach. Cardiovasc Intervent Radiol 2001; 24 : 154-60.

21. Noory E, Rastan A, Sixt S, et al. Arterial puncture closure using a clip device after transpopliteal retrograde approach for recanalization of the superficial femoral artery. J Endovasc Ther 2008; 15: 310-4.
22. Sidhu R, Pigott J, Pigott M, et al. Subintimal angioplasty for advanced lower extremity ischemia due to TASC II C and D lesions of the superficial femoral artery. Vasc Endovascular Surg 2010; 44: 633-7.

23. Kim SJ, Kim W, Kim JB, et al. Determinants of procedural success and patency following subintimal angioplasty in patients with TASC C and D femoropopliteal arterial disease. Circ J 2010; 74 : 1959-64.

24. El-Maadawy M, Rahim A, Abdelrahaman A. Retrograde transpopliteal angioplasty for superficial femoral artery occlusion. Technical point of view. Middle-East J Sci Res 2015; 23: 1470-4.

25. Beckman J. Peripheral endovascular revascularization some proof in the pudding? Circulation 2007; 115: 550-2.

26. Hirsch AT, Haskal ZJ, Hertzer NR, et al. ACC/AHA 2005 Practice Guidelines for the management of patients with peripheral arterial disease (lower extremity, renal, mesenteric, and abdominal aortic): a collaborative report from the American Association for Vascular Surgery/Society for Vascular Surgery, Society for Cardiovascular Angiography and Interventions, Society for Vascular Medicine and Biology, Society of Interventional Radiology, and the ACC/AHA Task Force on Practice Guidelines (Writing Committee to Develop Guidelines for the Management of Patients With Peripheral Arterial Disease): endorsed by the American Association of Cardiovascular and Pulmonary Rehabilitation; National Heart, Lung, and Blood Institute; Society for Vascular Nursing; TransAtlantic Inter-Society Consensus; and Vascular Disease Foundation. Circulation. 2006; 113: e463-654.

27. Amoroso NS, Shah S, Liou M, et al. Superficial femoral artery intervention by single transpedal arterial access. J Invasive Cardiol 2015; 27: E236-41.

28. Ruzsa Z, Nemes B, Bánsághi Z, et al. Transpedal access after failed anterograde recanalization of complex below-the-knee and femoropoliteal occlusions in critical limb ischemia. Catheter Cardiovasc Interv 2014; 83: 997-1007.

29. Health Quality Ontario. Stenting for peripheral artery disease of the lower extremities: an evidence-based analysis. Ont Health Technol Assess Ser 2010; 10: 1-88. 